\title{
ASK1-dependent endothelial cell activation is critical in ovarian cancer growth and metastasis
}

\author{
Mingzhu Yin, ${ }^{1}$ Huanjiao Jenny Zhou, ${ }^{1}$ Jiqin Zhang, ${ }^{1,2}$ Caixia Lin, ${ }^{2}$ Hongmei Li, ${ }^{1} \mathrm{Xia} \mathrm{Li},{ }^{1}$ Yonghao $\mathrm{Li},{ }^{3}$ \\ Haifeng Zhang, ${ }^{1}$ David G. Breckenridge, ${ }^{4}$ Weidong Ji, ${ }^{2}$ and Wang Min ${ }^{1,2}$ \\ 'Department of Pathology and the Vascular Biology and Therapeutics Program, Yale University School of Medicine, New \\ Haven, Connecticut, USA. ${ }^{2}$ Center for Translational Medicine, The First Affiliated Hospital, and ${ }^{3}$ Zhongshan Ophthalmology \\ Hospital, Sun Yat-sen University, Guangzhou, China. ${ }^{4}$ Cilead Sciences Inc., Foster City, California, USA.
}

\begin{abstract}
We have recently reported that tumor-associated macrophages (TAMs) promote early transcoelomic metastasis of ovarian cancer by facilitating TAM-ovarian cancer cell spheroid formation. ASK1 is known to be important for macrophage activation and inflammation-mediated tumorigenesis. In the present study, we show that ASK1 deficiency attenuates TAM-spheroid formation and ovarian cancer progression in an orthotopic ovarian cancer model. Interestingly, ASK1 in stroma, but not in TAMs, is critical for peritoneal tumor growth of ovarian cancer. Moreover, overexpression of an ASK1 inhibitory protein (suppressor of cytokine signaling-1; SOCS1) in vascular endothelium attenuates vascular permeability, TAM infiltration, and ovarian cancer growth. Mechanistically, we show that ASK1 mediates degradation of endothelial junction protein VE-cadherin via a lysosomal pathway to promote macrophage transmigration. Importantly, a pharmacological ASK1 inhibitor prevents tumor-induced vascular leakage, macrophage infiltration, and tumor growth in two mouse models. Since transcoelomic metastasis is also associated with many other cancers, such as pancreatic and colon cancers, our study provides ASK1 as a therapeutic target for the treatment of ovarian cancer and other transcoelomic metastasis cancers.
\end{abstract}

Conflict of interest: The authors have declared that no conflict of interest exists.

Submitted: November 17, 2016 Accepted: August 11, 2017 Published: September 21, 2017

Reference information: JCI Insight. 2017;2(18):e91828. https://doi.org/10.1172/jci. insight. 91828 .

\section{Introduction}

Macrophages are a major cell type in animal and human tumors, where they are commonly termed tumor-associated macrophages (TAMs). Many studies have suggested that TAMs act as a source of local and systemic cues to support the proliferation, survival, and migration of tumor cells $(1,2)$. It has been proposed that TAMs are predominantly polarized in the tumor microenvironment toward an M2-like subtype and that this underlies their ability to promote the tumor growth and angiogenesis (3). This is also supported by clinical studies showing the predictive value of TAMs for patient survival $(4,5)$. Furthermore, this array of tumor-promoting functions is consistent with clinical studies showing high macrophage density in many human cancer types to be associated with poor prognosis. TAMs not only enhance tumor growth and progression, but they also modulate the efficacy of suppressing antitumor immunity by preventing activation of DCs, cytotoxic T lymphocytes (CTLs), and NK cells; they facilitate tumor regrowth, revascularization, and spread after the treatment $(6,7)$. Therefore, attempts are now being made to either target key molecules that regulate their recruitments into tumors or reeducate these cells toward a cytotoxic M1-like phenotype.

Apoptosis signal-regulating kinase 1 (ASK1), a member of the MAP3K family activating MAP2K$\mathrm{JNK} / \mathrm{p} 38$ cascades, is a key kinase involved in stress-induced apoptosis or nonapoptotic death (8-10). Activation of ASK1 leads to death in many cell types, including tumor cells. Thus, ASK1 is a central target of many cellular survival factors, including Raf-1, Akt, and hsp90 (11-13). It is expected that ASK1 functions as a tumor suppressor gene in cancer cells and is a potential therapeutic target for anticancer drugs. Indeed, downregulation of ASK1 activity has been detected in several malignant cancers and associated proliferation of cancer cells $(14,15)$. Conversely, anticancer therapeutics, including radiotherapy and chemotherapy, can induce cancer cell apoptosis by activating ASK1 (16). We have also demonstrated that an anticancer prodrug Laromustine derivative methyl isocyanate activates ASK1 and leads to the nonapoptotic 
death of endothelial cells (ECs) and inhibition of tumor angiogenesis (17). On the other hand, ASK1 is also critical in inflammation by promoting cytokine production in immune cells (primarily macrophages) and tumor cells, and by mediating cytokine responses in vascular ECs $(18,19)$. Consistently, it has been reported that ASK1-dependent cytokine production in inflammatory cells is required for tumor promotion in a 12-O-tetradecanoylphorbol-13-acetate-induced (TPA-induced) skin tumorigenesis model (14). In this model, a potent tumor promoter, TPA, induces hyperplasia with infiltration of inflammatory cells; TPA-induced infiltration of immune cells, production of cytokines, and skin hyperplasia are reduced in ASK1-KO mice. However, the role of ASK1 in tumor microenvironment has not been directly examined. ASK1 can be activated in response to proinflammatory cytokines. Studies in in vitro systems and from ASK1-KO mice have shown that ASK1 is a critical mediator in cytokine (e.g., TNF-induced) inflammatory signaling $(9,20,21)$. ASK1 is critical for lipopolysaccharide-induced (LPS-induced) p38 activation and cytokine production in splenocytes and macrophages (20). In the vasculature, ASK1 mediates EC activation and inflammation (19). Based on these studies, it is conceivable that ASK1 in the tumor microenvironment (particularly in vascular EC and macrophages) promotes tumor growth and metastasis by mediating inflammatory responses $(14,15)$.

Ovarian cancer (OC) is the second most common gynecological cancer and is the most lethal gynecological malignancy worldwide $(22,23)$. The primary metastasis mode of OC is transcoelomic, which involves a tumor cell that is detached from the primary tumor and is transported and implanted in peritoneum and pelvic organs. In this process, detached tumor cells aggregate as spheroids within the abdominal cavity to overcome anoikis, immune attack, and chemotherapy (24-26). These spheroids can spread over the peritoneal cavity, invade the peritoneum, and implant in pelvic organs and omentum. Therefore, spheroid formation is a key step in transcoelomic metastasis. We have recently reported that TAMs in the peritoneal cavity promote tumor cell survival, proliferation, and early transcoelomic metastasis of OC by facilitating TAM-OC cell spheroid formation in orthpotopic OC models (27). Our initial objective was to investigate the role of ASK1 in TAM-promoted OC progression in an orthotopic OC model. Our present study reveals that ASK1-dependent EC activation and macrophage infiltration, a step prior to spheroid formation, play a critical role in OC growth and metastasis.

\section{Results}

Attenuated OC growth and peritoneal implantation in ASK1-KO mice. To determine the role of ASK1 in cancer growth, we applied an orthotopic mouse OC model to C57BL/6 (WT) and ASK1-KO female recipient mice by injecting $1 \times 10^{6}$ mouse ID8 cells i.p. Mouse body weights were measured from day 0-60 after tumor implantation. Ascitic fluid and tumor cells were harvested on day 60 before death of recipient mice was observed. Tumor growth, accumulation of ascitic fluid, and net tumor weights were drastically attenuated in ASK1-deficient mice compared with the WT group (Figure 1, A-C). Tumor implantations in omentum, peritoneum, mensentery, diaphragm, and pelvic cavity were examined under microscope and quantified. The results showed that tumor implantation in these tissues were also markedly reduced in ASK1-deficient mice compared with the WT group (Figure 1, D and E). We further measured mouse modality and survival rates, and ASK1 deficiency prolonged the survival of tumor-bearing mice (Figure 1F). These data suggest that a global deletion of ASK1 in mice attenuates OC growth and peritoneal implantation.

Attenuated peritoneal macrophage infiltration and spheroid formation in ASK1-KO mice. We have recently shown that the interactions between TAMs and ID8 cells promote spheroid formation during the process of OC transcoelomic seeding. In the orthotopic mouse OC model of WT and ASK1-deficient mice, total peritoneal cells were harvested at 8 weeks when all TAMs were M2-like subtype. Cells were immunostained with anti-F4/80, CD11b, and CD3e followed by FACS analyses. Results showed that the numbers of $\mathrm{F} 4 / 80^{+} \mathrm{CD} 11 \mathrm{~b}^{+}$, but not $\mathrm{CD} 3 \mathrm{e}^{+}$cells, were significantly reduced in ASK1-deficient mice compared with WT mice (Figure 2, A and B). Immunostaining with CD68 of spheroids from ascites showed that the size of tumor spheroids and $\mathrm{CD} 68^{+}$macrophages inside the spheroids were significantly decreased in ASK1-deficient mice (Figure 2, C-E). Similarly, $\mathrm{CD}^{+} 8^{+}$macrophages inside the peritoneal implantations were also reduced in ASK1-deficient mice (Supplemental Figure 1; supplemental material available online with this article; https://doi.org/10.1172/jci.insight.91828DS1).

We have recently demonstrated that TAMs are polarized to M2-like subtype in the peritoneal cavity microenvironment during OC progression. Specifically, the initial infiltrated macrophages (at 1 week) induced by ovarian tumor cells strongly expressed M1-like marker genes (Ly6G/C, CCR2, IFN $\alpha$ R, iNOS). However, infiltrated macrophages gradually gain expression of M2-like marker genes (CD206 [mannose 
A

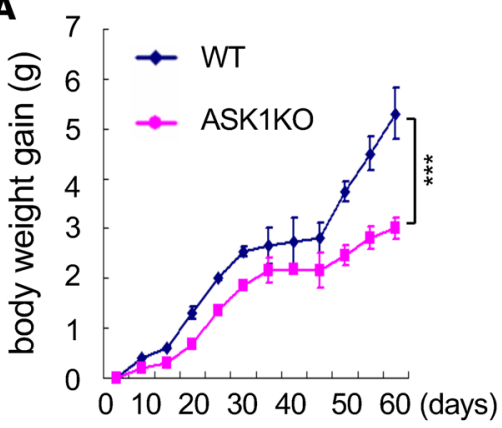

B

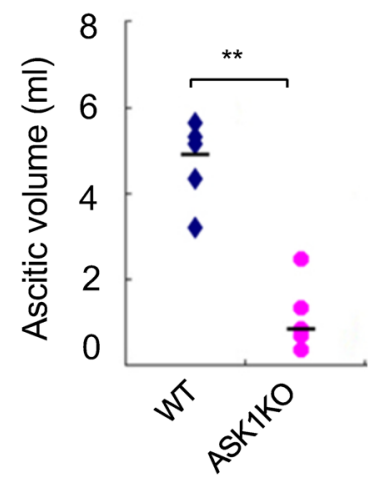

C

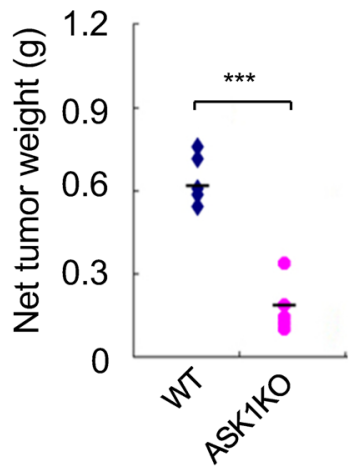

D
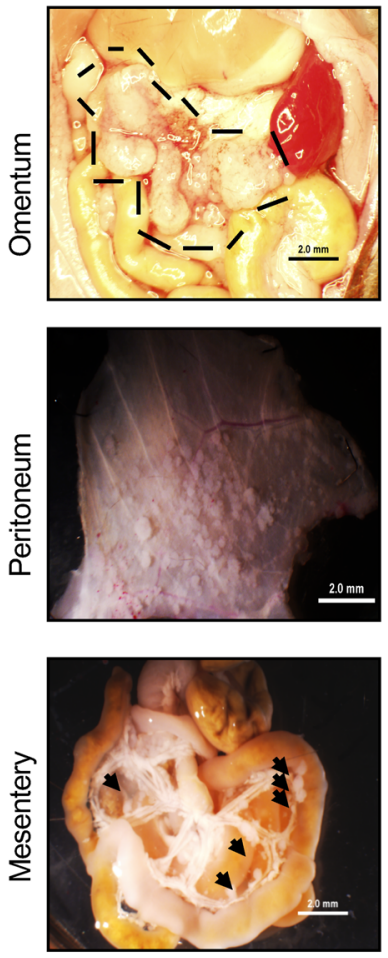

ASK1KO
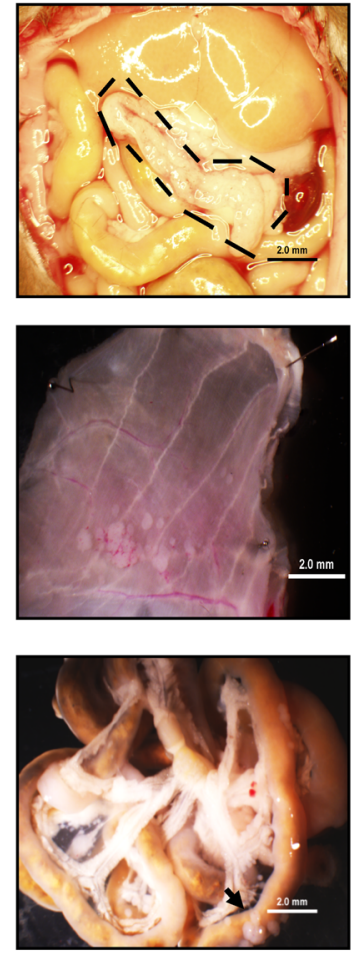

E

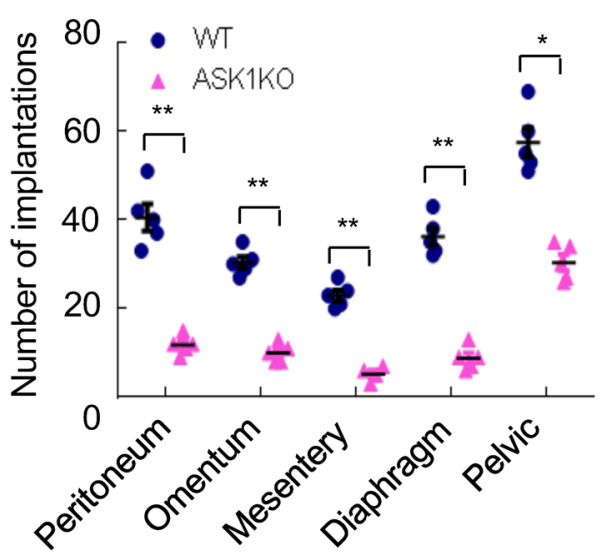

$\mathbf{F}$

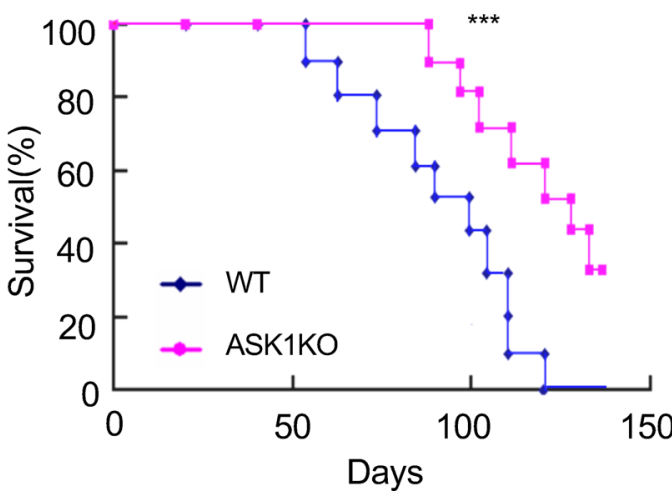

Figure 1. Attenuated ovarian cancer growth and peritoneal implantation in ASK1-KO mice. An orthotopic mouse ovarian cancer model was established by injecting $1 \times 10^{6}$ mouse ID8 cells i.p. to C57BL/6 (WT) and ASK1-KO female recipient mice. (A) Mouse body weights were measured at indicated time points (day 0-60), and body weight gains are shown. (B and C) Ascitic fluid volumes and net tumor weights were measured at day 60 . Data in A-C are presented as means \pm SEM, $n=10 .{ }^{* *} P<0.01$; ${ }^{* *} P<0.001$ (two-sided student's $t$ test). (D) Representative images of tumor implantations in omentum, peritoneum, and mesentery at day 60 . Scale bars: $2 \mathrm{~mm}$. Tumor implantations are indicated by outlines and arrows. (E) Statistical analyses of tumor implantations in omentum, peritoneum, mesentery, diaphragm, and pelvic cavity. $n=5$. Data are presented as means $\pm S E M .{ }^{*} P<0.05 ;{ }^{* *} P<0.01$ (two-sided student's $t$ test). (F) Mouse modality was monitored, and survival rates were quantified; $n=24$ mice per group. Kaplan-Meier analyses using the log-rank test was performed; ${ }^{* * *} P<0.001$.

receptor], CX3CR1, Arginase 1, and CD163 [scavenger receptor cysteine-rich type 1 protein M130]) during tumor progression (4-8 weeks). To determine if ASK1 deficiency affects TAM polarization in the OC mod$\mathrm{el}, \mathrm{F} 4 / 80^{+} \mathrm{CD} 11 \mathrm{~b}^{+}$macrophages were harvested at 8 weeks after tumor injection, and a set of M1 subtypespecific and M2 subtype-specific markers were examined by quantitative reverse transcription PCR (qRTPCR). The results indicated that TAM polarization was not dramatically altered in ASK1-deficient mice (Figure 2F). Taken together, these data suggest that ASK1 deficiency attenuates peritoneal macrophage infiltration and TAM-tumor cell spheroid formation, but not subsequent polarization of TAMs, leading to reduced cancer growth and peritoneal implantation in the ID8 OC mouse model. 
A
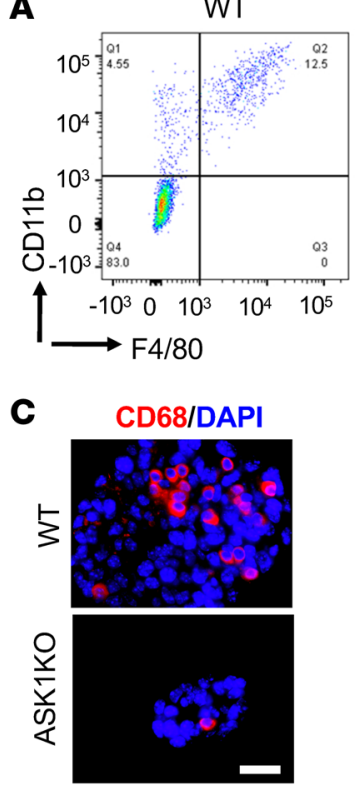

\section{$\mathbf{F}$} - Monocyte a TAM (WT) ^ TAM (KO)
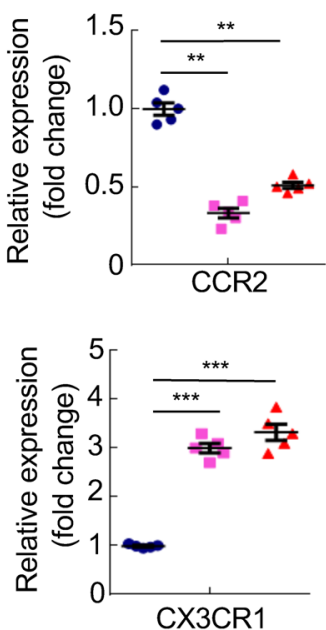
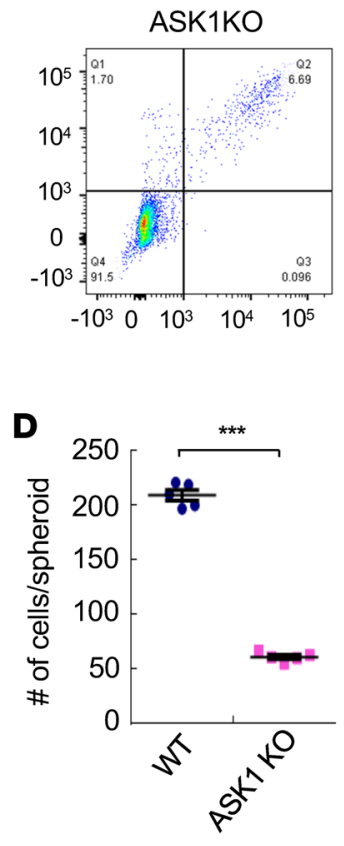

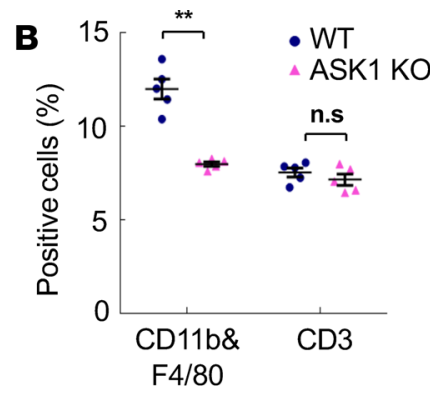

E

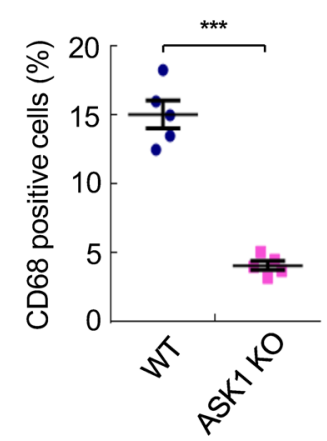

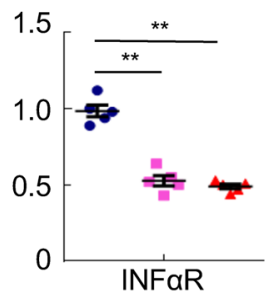
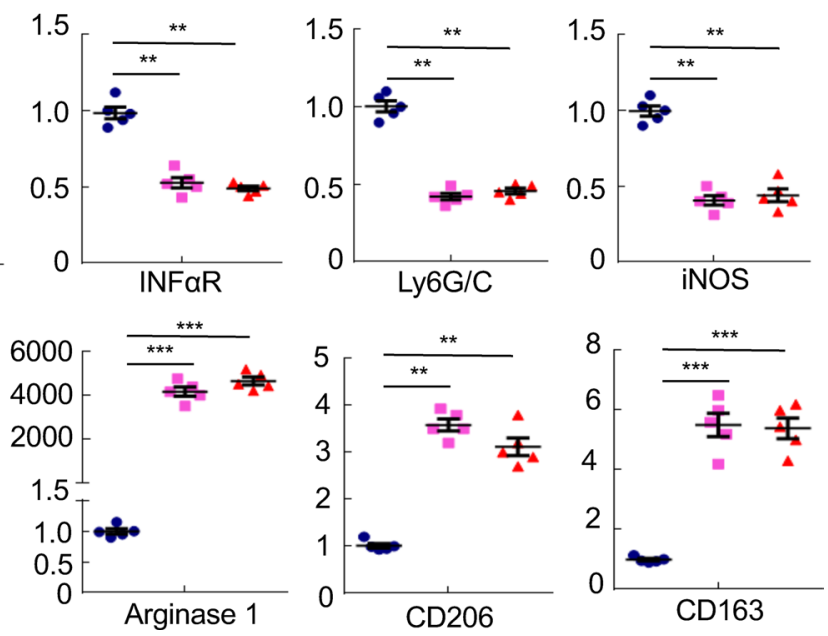

Figure 2. Attenuated peritoneal macrophage infiltration and spheroid formation in ASK1-KO mice. In the orthotopic mouse OC model, total peritoneal cells were harvested at day 60 of WT and ASK1-KO female recipient mice. (A and $\mathbf{B})$ Cells were immunostained with anti-F4/80, CD11b, and CD3e followed by FACS analyses. Isotype IgGs were used as controls. Representative images are shown in $\mathbf{A}$. Percentage of $\mathrm{F} 4 / 80^{+} \mathrm{CD} 11 \mathrm{~b}^{+}$and $\mathrm{CD}^{\mathrm{C}} \mathrm{e}^{+}$cells in peritoneal implantations were quantified in B. Data are presented as means \pm SEM, $n=5$. ${ }^{* *} P<0.01$ (two-sided student's $t$ test). (C-E) Spheroids from ascites were collected at day 60 and mounted on slides followed by immunostaining with CD68 (C). Scale bar: $20 \mu \mathrm{m}$. Spheroid sizes (D) and \% of CD68 ${ }^{+}$cells within spheroids (E) were quantified. (F) Accumulation of M2-like subtype tumor-associated macrophages (TAMs) had no difference between WT and ASK1-KO tumor models. F4/80+ CD11b+ macrophages in the orthotopic implantation tumor were harvested from WT and ASK1-KO mice at 8 weeks after i.p. injection of ID8 cells. M1 subtype-specific and M2 subtype-specific markers were determined by qRT-PCR. Peripheral blood monocytes were used as a control. All data are presented as means $\pm \mathrm{SEM}, n=5 .{ }^{*} P<0.01$; ${ }^{* * *} P<0.001$ (two-sided student's $t$ test with no correction for multiple comparisons) compared with gene expressions in week-1 individual cells.

ASK1 in stroma but not in TAMs is critical for peritoneal tumor growth of OC. Since ASK1$\mathrm{KO}$ was a global deletion of ASK1, we determined the role of ASK1 in TAMs by peritoneal transplantation method that we recently described (27). Twenty days after ID8 tumor injection in the orthotopic mouse OC model, half of the WT recipient mice received $1 \times 10^{6}$ $\mathrm{F} 4 / 80^{+} \mathrm{CD} 206^{+} \mathrm{TAM}$ isolated from OC-bearing WT or ASK1-KO donor mice. Because ASK1-KO donor mice had much fewer TAMs than WT donor mice, we used more ASK1-KO mice to obtain a similar number of F4/80 ${ }^{+} \mathrm{CD} 206^{+}$TAMs. Surprisingly, TAMs from both WT and ASK1-KO donor mice similarly augmented tumor growth, accumulation of ascitic fluid, and net tumor weight, as well as tumor implantations in WT recipient mice (Figure 3, A-D). These data suggest that ASK1 signaling in TAMs is not critical for TAM-promoted peritoneal tumor growth. We reasoned that ASK1-KO TAMs also promote tumor growth once implanted into the peritoneal cavity, even in ASK1-KO recipient mice. We detected increased $\mathrm{F} 4 / 80^{+} \mathrm{CD} 11 \mathrm{~b}^{+}$macrophages, but not $\mathrm{T}$ cells, in the peritoneal cavity of ASK1-KO recipient mice (Supplemental Figure 2, A and B). As we have demonstrated for WT recipient mice, TAMs significantly augmented tumor growth, accumulation of ascitic fluid, and net tumor weight, as well as tumor implantations in ASK1-KO recipient mice (Figure 3, E-H). Similarly, the size of tumor spheroids and $\mathrm{CD} 8^{+}$macrophages inside the spheroids were significantly increased by TAM transplantation in ASK1-deficient mice (Supplemental Figure 2, C-E). Accordingly, mouse survival rate was greatly decreased by TAMs (Supplemental Figure 2F). Again, WT and ASK1-deficient TAMs exhibited similar effects in ASK1-KO recipient mice. These data suggest that ASK1 in stroma, but not in TAMs, is critical for peritoneal tumor growth of OC. Given that macrophage infiltration was markedly reduced in ASK1KO recipient mice, ASK1 likely plays a critical role in regulating macrophage infiltration into the peritoneal cavity, leading to reduced numbers of macrophages and OC growth in ASK1-KO mice. 
A

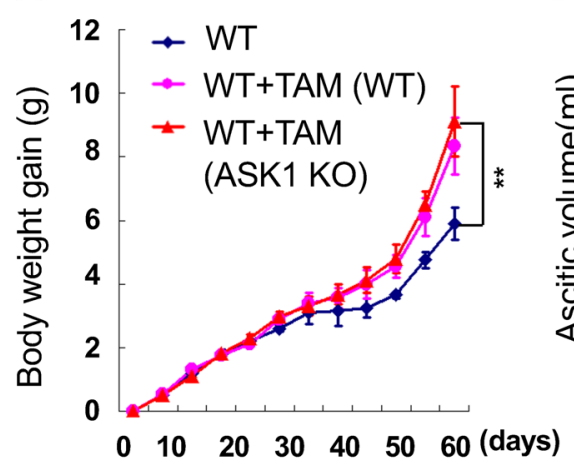

B

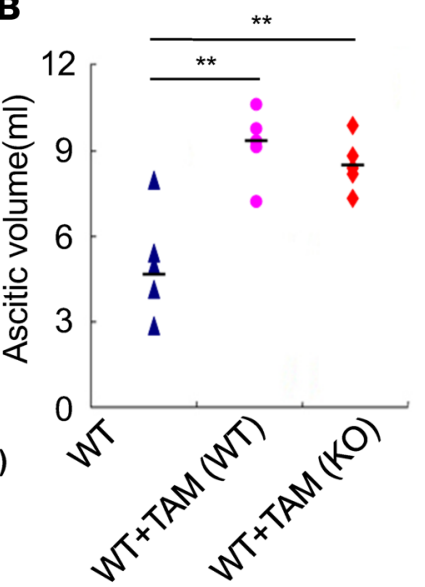

C

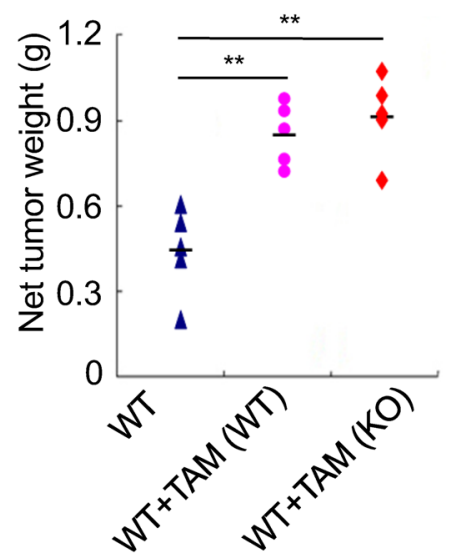

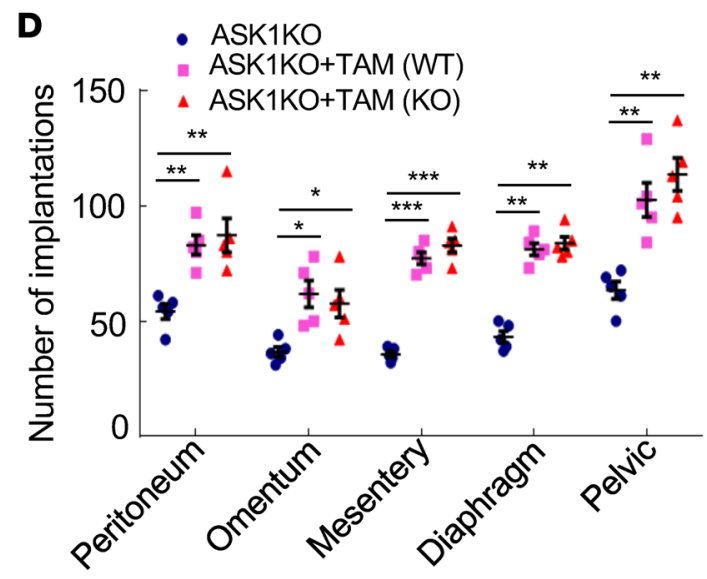

E

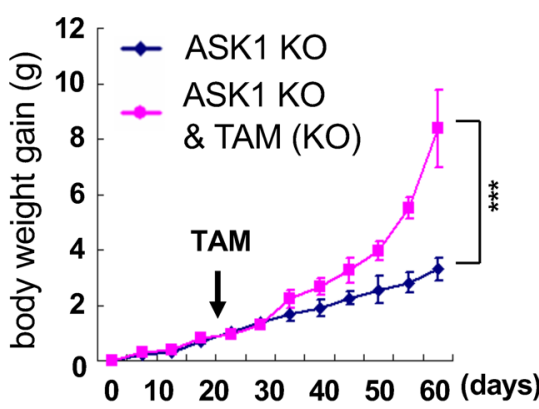

$\mathbf{F}$

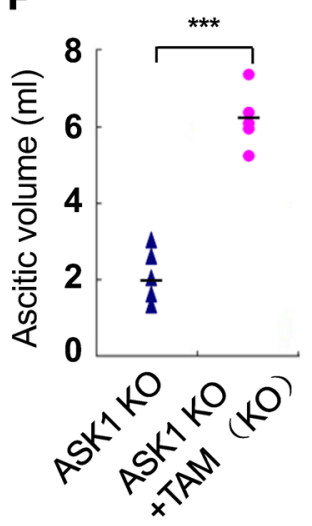

G

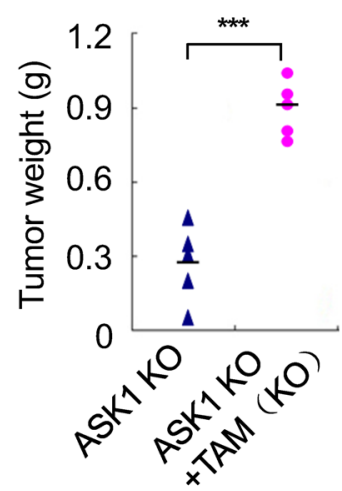

- ASK1KO

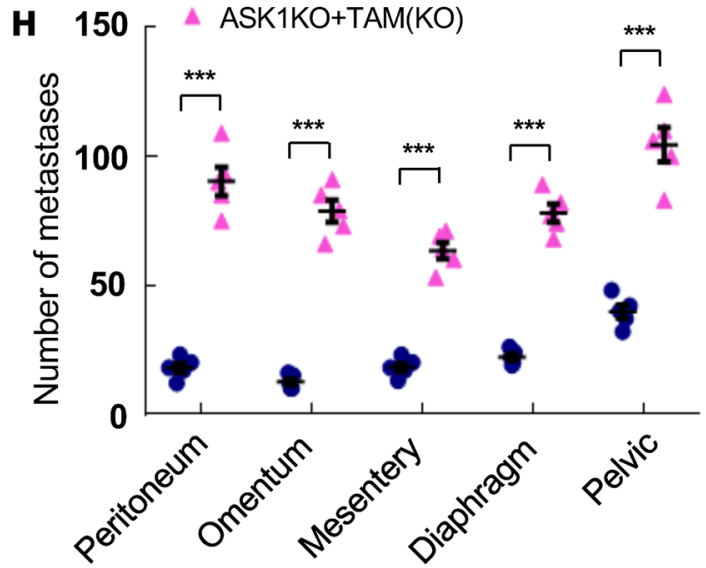

Figure 3. ASK1 in stroma but not in TAMs is critical for peritoneal tumor growth of ovarian cancer. Twenty days after ID8 tumor injection in the orthotopic mouse ovarian cancer model, half of the recipient mice received $1 \times 10^{6} \mathrm{~F} 4 / 80^{+} \mathrm{CD} 206^{+}$tumor-associated macrophages (TAMs) isolated from ovarian cancer-bearing donor mice. (A-D) Effects of WT TAMs on tumor growth in ASK1-KO recipient mice. (E-H) Effects of WT and ASK1-KO TAMs on tumor growth in WT recipient mice. Mouse body weights (A and $\mathbf{E}$ ), ascitic fluid volumes (B and $\mathbf{F}$ ), net tumor weights ( $\mathbf{C}$ and $\mathbf{G}$ ), and tumor implantations $(\mathbf{D}$ and $\mathbf{H})$ were measured at day 60. Data are presented as means \pm SEM, $n=10$. ${ }^{*} P<0.05$; ${ }^{*} P<0.01 ;{ }^{* *} P<0.001$ (two-sided student's $t$ test with no correction for multiple comparisons).

EC expression of an ASK1 inhibitory protein attenuates OC growth. Macrophage infiltration into the peritoneal cavity can be regulated by vascular endothelium in peritoneum, tumor cell-secreted chemokines, and macrophage-intrinsic migratory properties $(1,2)$. To directly determine the role of ASK1 in vascular endothelium, we applied the orthotopic OC model to VESOCS1 mice, a transgenic line expressing suppressor of cytokine signaling-1 (SOCS1) specifically in vascular endothelium. SOCS is a cytokine-inducible negative regulator of cytokine signaling that suppresses ASK1 signaling by promoting ASK1 degradation 
A

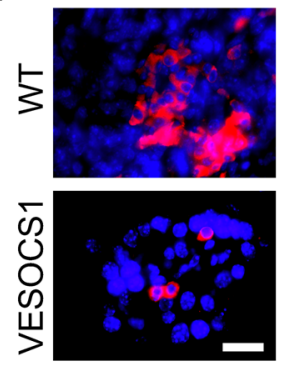

B

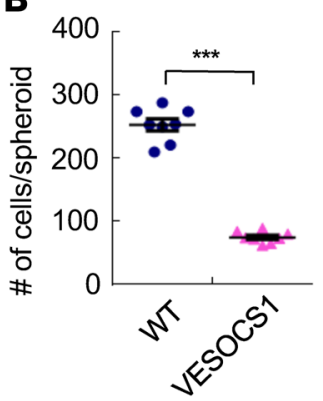

C

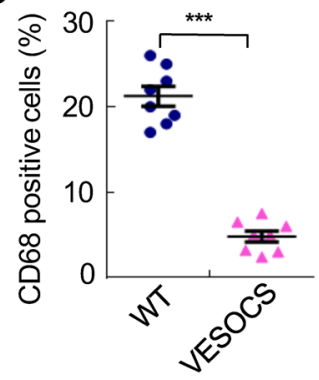

D

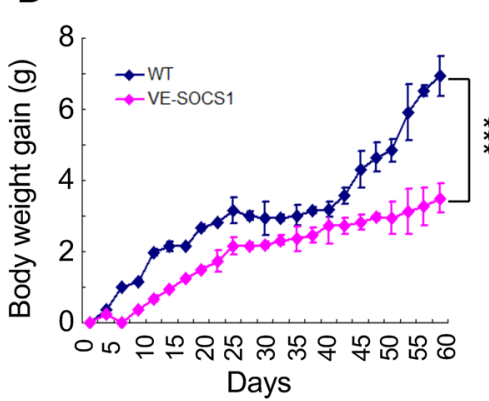

G

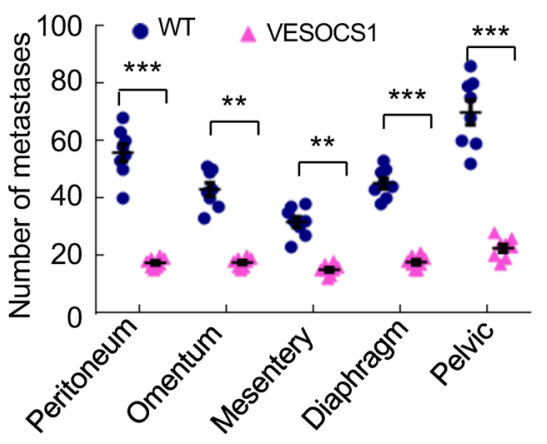

E

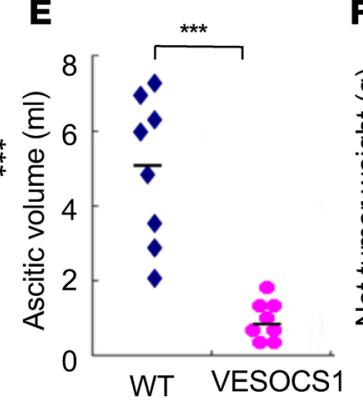

H

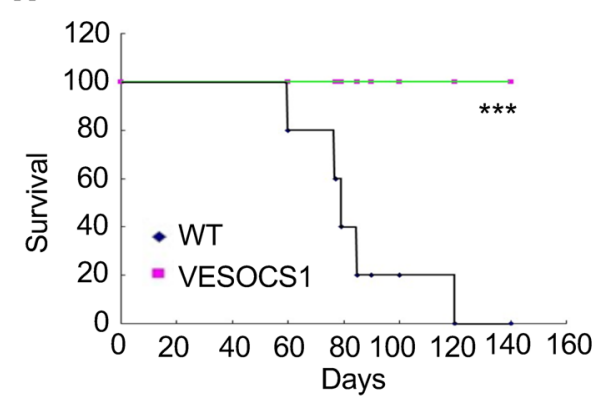

Figure 4. A transgene of ASK1 inhibitor SOCS1 in vascular endothelium attenuates ovarian cancer growth. Vascular endothelial transgenic mice expressing suppressor of cytokine signaling (VESOCS1) and nontransgenic WT mice were injected with ID8 cells. (A-C) Spheroids from ascites were collected at day 60 and mounted on slides. Spheroids were examined by CD68 staining (A). Scale bar: 20 $\mu \mathrm{m}$. Spheroid sizes (B) and \% of CD68+ cells within spheroids (C) were quantified. Data are presented as means $\pm \mathrm{SEM}, n=10$. ${ }^{* * *} P<$ 0.001 (two-sided student's $t$ test). (D) Mouse body weights were measured at indicated time points (day 0-60), and body weight gains are shown. (E and F) Ascitic fluid volumes and net tumor weights were measured at day 60. (G) Statistical analyses of tumor implantations in omentum, peritoneum, mensentery, diaphragm, and pelvic cavity, $n=5$. Data in $\mathbf{A}-\mathbf{G}$ are presented as means \pm SEM. ${ }^{*} P<0.01$; ${ }^{* * *} P<0.001$ (two-sided student's $t$ test). (H) Mouse modality was monitored, and survival rates were quantified; $n=24$ mice per group. Kaplan-Meier analyses using the log-rank test was performed. ${ }^{* *} P<0.001$.

$(28,29)$. Previously, we have shown that macrophage infiltration into aortic neointima is attenuated in a graft arteriosclerosis model in SOCS1 transgenic mice $(30,31)$. To exclude the possibility that the number of monocytes is inherently reduced in VESOCS1 as compared with WT mice, we examined CD11b ${ }^{+}$and $\mathrm{CD} \mathrm{e}^{+}$cells in BM and spleen by immunostaining and FACS analyses. The results showed that there was no significant difference in the number of macrophages and $\mathrm{T}$ cells in BM, spleen, or peripheral blood between VESOCS1 and WT mice under physiological conditions (Supplemental Figure 3, A-E). We then determined if inflammation-induced macrophage infiltration into the peritoneal cavity is attenuated in VESOCS1 mice by injecting 4\% thioglycollate i.p. (32) (Supplemental Figure 4, A and B). We observed that the number of total inflammatory cells and $\mathrm{F} 4 / 80^{+}$cells were significantly less in peritoneal lavage fluid of VESOCS1 mice, as compared with WT mice, by cell counting and immunstaining (Supplemental Figure 4, B-E). These results suggested that the endothelial-specific SOCS1 transgene does not alter the total number of macrophages, but inhibits inflammation-induced peritoneal transmigration of macrophages.

We next determined macrophage infiltration in the orthotopic OC model using VESOCS1 and nontransgenic WT mice. Total peritoneal cells were harvested at 8 weeks after ID8 cell injection. As we observed in ASK1-KO mice, the number of $\mathrm{F} 4 / 80^{+} \mathrm{CD} 11 \mathrm{~b}^{+}$macrophages were significantly reduced in VESOCS1 mice compared with WT mice (data not shown). Accordingly, the size of tumor spheroids and $\mathrm{CD}^{+} 8^{+}$macrophages inside the spheroids were significantly decreased in VESOCS1 mice (Figure 4, A-C). Consistent with reduced TAM-associated spheroid formation, the SOCS1 transgene in the endothelium strongly attenuated tumor growth, accumulation of ascitic fluid, and net tumor weights as well as tumor implantations, compared with the WT group (Figure 4, D-G). The VESOCS1 transgene also prolonged 
A

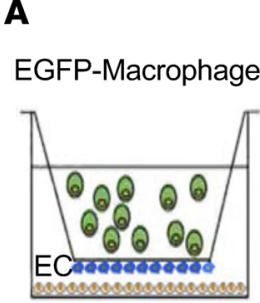

Tumor cell
B

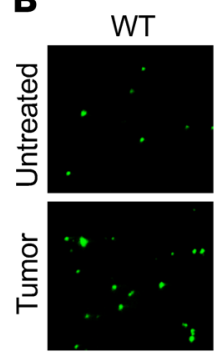

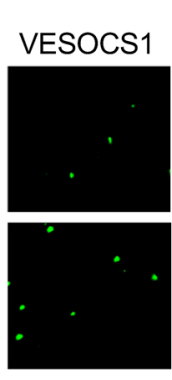

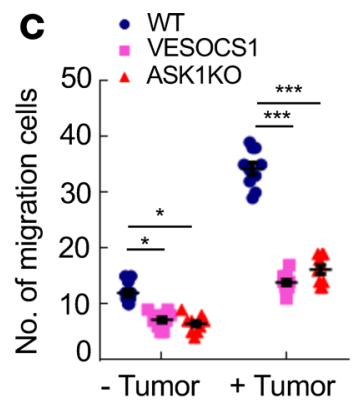

Figure 5. The SOCS1-ASK1 axis regulates EC permeability and macrophage transmigration by regulating EC junctions.

(A-C) Vascular endothelial transgenic mice expressing suppressor of cytokine signaling (VESOCS1) or ASK1 deficiency attenuated macrophage-endothelial transmigration. Isolated WT and VESOCS1 mouse lung microvascular ECs (MLMVEC) were seeded onto the surface of collagen gel in the absence or presence of ID8 cells at the bottom for 12 hours. GFP-expressing macrophages were loaded onto the mouse EC monolayer for 24 hours (A). Transendothelial migrated GFP+ macrophages were visualized by immunofluorescence microscopy. Representative images are shown in B. Magnification, 100x. Quantifications of transmigrated cells are shown in C. Data are expressed as mean \pm SEM, $n$ $=10 .{ }^{*} P<0.005,{ }^{* *} P<0.001$ (two-tailed student's $t$ test. (D-F) Immunostaining of VE-cadherin in MLMVECs isolated from WT, VESOCS1, and ASK1-KO mice. Scale bar: $25 \mu \mathrm{m}$. (D and E) Representative images are shown. (F) Quantifications of VE-cadherin immunofluorescence intensity. Data are expressed as mean \pm SEM, $n=10 .{ }^{* *} P<0.001$ (two-sided student's $t$ test with no correction for multiple comparisons). (G-J) Expression of junctional proteins in WT, ASK1-KO, and VESOSC1 MLMVECs by Western blotting ( $\mathbf{G}$ and $\mathbf{~}$ ). Quantitation of blot by densitometry (H and J). Data are expressed as mean $\pm \mathrm{SEM}$, $n=3 .{ }^{*} P<0.005$; ${ }^{*} P<0.01 ;{ }^{* *} P<0.001$. Two-sided student's $t$ test with no correction for multiple comparisons.

G

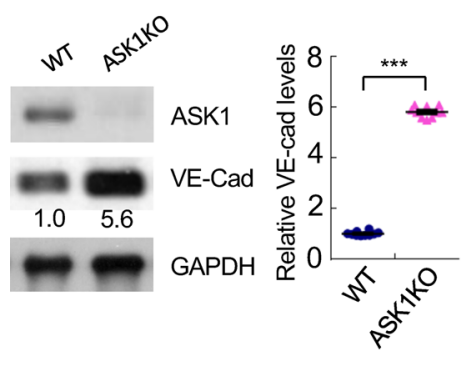

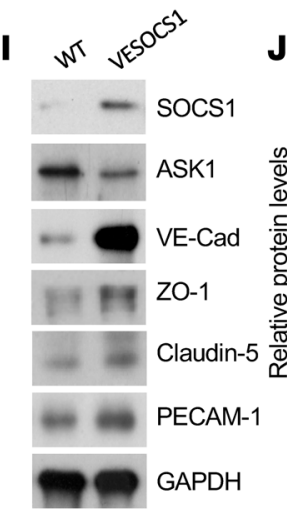

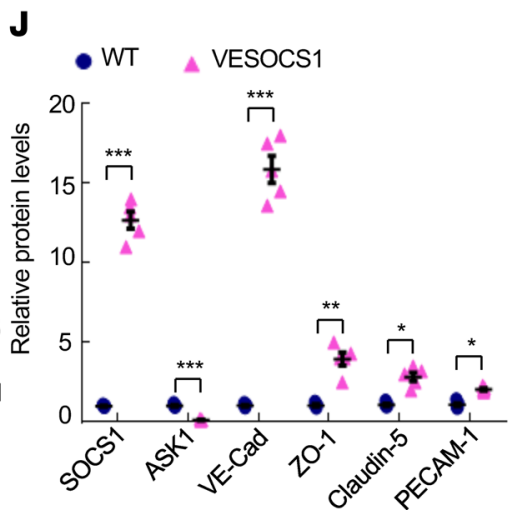

the survival of tumor-bearing mice (Figure 4H). These data suggest that endothelial expression of ASK1 inhibitor SOCS1 attenuates macrophage infiltration, OC growth, and peritoneal implantations.

The SOCS1-ASK1 axis regulates EC permeability and macrophage transmigration by regulating EC junctions. To explore the mechanism by which the SOCS1-ASK1 axis regulates tumor cell-induced monocyte transmigration, we employed a transwell assay in which PE-ID8 tumor cells (isolated from tumor-bearing mice) at the bottom chamber induce transmigration of EGFP-labeled monocytes at the top chamber across a cultured EC monolayer (Figure 5A). VE-SOCS1 or ASK1-KO ECs significantly inhibited EGFP ${ }^{+}$monocyte transmigration cross the cultured EC layers (Figure 5, B and C). We reasoned that peritoneal microvessel permeability plays an important role in the process of OC implantation metastasis through regulating macrophage infiltration. We first detected ASK1 expression in the peritoneum vessels exhibiting colocalization with EC marker CD31 (Supplemental Figure 5, A and B). Tumor cells in the peritoneal cavity induced dilation of microvessels concomitant with disruptions of endothelial junctions, as visualized by whole mount immunostaining of VE-cadherin in peritoneal vessels (Supplemental Figure 5, C and D). Moreover, we observed an augmented immunostaining of VE-cadherin, but not CD31 (PECAM-1), in the VESOCS1 peritoneum by whole mount immunostaining (Figure 5D). We observed a similar pattern of increased VE-cadherin immunostaining in isolated microvascular ECs from VESOCS1 and ASK1-KO mice (Figure 5, E and F). 
A

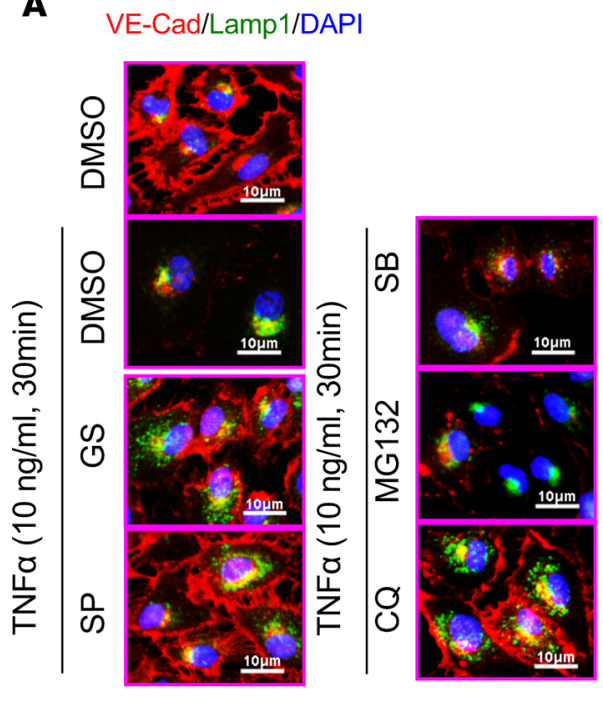

B

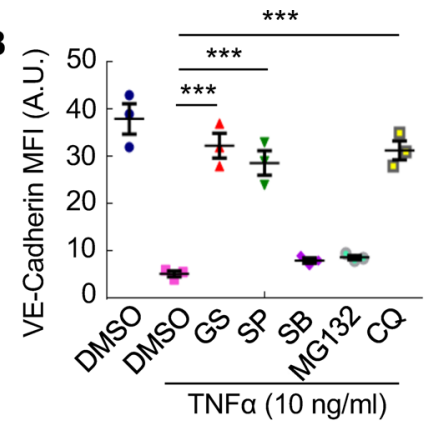

C

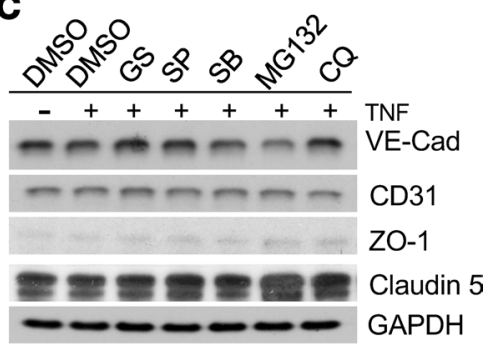

E

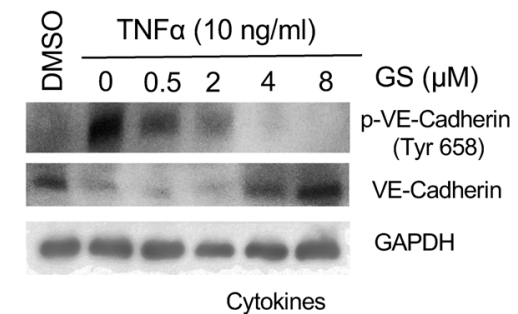

$\mathbf{G}$

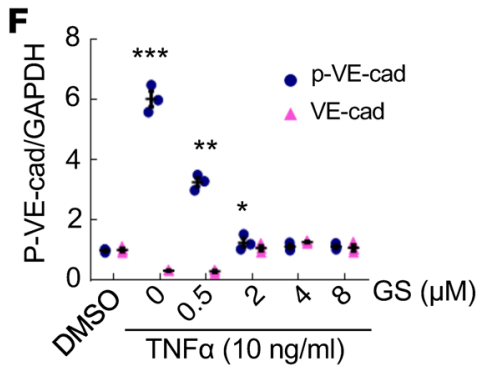

Figure 6. ASK1 mediates VE-cadherin degradation via a lysosomal pathway. Human lung microvessel ECs (HLMVEC) were untreated or treated with TNF ( $10 \mathrm{ng} / \mathrm{ml}$ for $30 \mathrm{~min}$ ) in the absence or presence of various inhibitors of ASK1, JNK, p38, MG132, and chloroquin as indicated. (A) Coimmunofluorescent staining of VE-cadherin (red) and late endosome marker Lamp1 (green). Representative images are shown. Scale bars: $10 \mu \mathrm{m}$. (B) Quantifications of junctional VE-cadherin. Data are expressed as mean \pm SEM, $n=30$ (10 images from each experiment). ${ }^{* *} P<0.001$. (C and D) VE-cadherin protein levels were determined by Western blotting (C). Relative VE-cadherin levels were quantified by densitometry with normalization of untreated group (D). ( $E$ and F) HLMVEC were untreated or treated with TNF (10 $\mathrm{ng} / \mathrm{ml}$ for $30 \mathrm{~min}$ ) in the absence or presence of various concentrations of ASK1. VE-cadherin and $\mathrm{p}-\mathrm{VE}$-cadherin protein levels were determined by Western blotting (E). Relative VE-cadherin and pVE-cadherin levels were quantified by densitometry with normalization of untreated group $(\mathbf{F})$. Data are expressed as mean $\pm \mathrm{SEM}, n=3 .{ }^{*} P<0.01$ (two-sided student's $t$ test with no correction for multiple comparisons). (C) Schematics of ASK1JNK-mediated VE-cadherin lysosomal-dependent degradation in ECs. TNF- $\alpha$ activates ASK1-JNK signaling and enhances phosphorylation and degradation of VE-cadherin through the lysosomal pathway in ECs. Inhibition of ASK1 (CS444217), JNK (SP600125), and lysosome (chloroquine) prevents VE-cadherin phosphorylation and degradation.

We also found that VE-cadherin was drastically enhanced in both ASK1-KO and VESOCS1 microvascular ECs by Western blotting (Figure 5, G-J). Of note, ASK1 expression was markedly reduced in VESOCS1 ECs, consistent with our previous findings that SOCS1 directs ASK1 degradation in vascular ECs $(28,29)$. Other junctional proteins (ZO-1, claudin-5) and adhesion molecule PECAM-1 were only weakly increased in VESOCS1 microvascular ECs (Figure 5, I and J). Taken together, these data demonstrated that SOCS1 and ASK1 regulate EC permeability and macrophage transmigration by regulating EC junctions.

ASK1 mediates cytokine-induced VE-cadherin degradation via a lysosomal pathway. To define the molecular mechanism by which ASK1 mediates VE-cadherin junctions, we reasoned that tumor cells in the peritoneal cavity provide proinflammatory cytokines that activate ASK1 and induce peritoneal microvessel permeability and macrophage transmigration. We examined gene expression of a panel of cytokines by qRT-PCR in naive ID8 and PE-ID8 ovarian tumor cells isolated from tumor-bearing mice. We found that proinflammatory cytokines (IL-1 $\beta$, IL-6, INF- $\alpha$, INF- $\gamma$, and TNF- $\alpha$ ) increased in PE-ID8 cells with TNF- $\alpha$ being induced the most (Supplemental Figure 6A). Indeed, condition media from PE-ID8 more strongly induced disruptions of EC junctions upon exposure to cultured mouse lung microvascular endothelial cells (MLMVEC) (Supplemental Figure 6B). TNF was detected in both ascites and condition media from PE-ID8 (Supplemental Figure 6, 


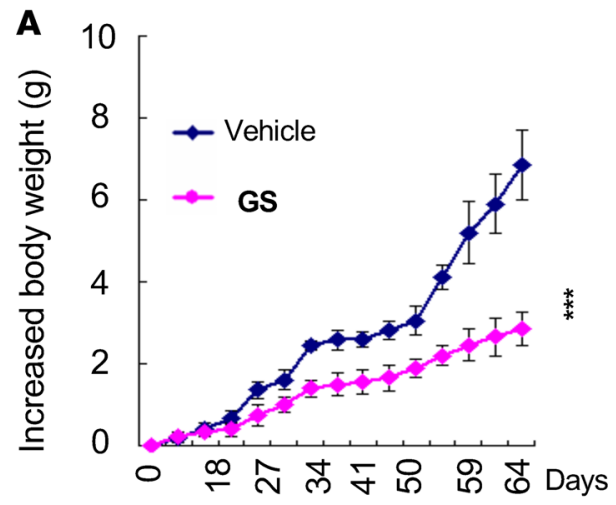

D

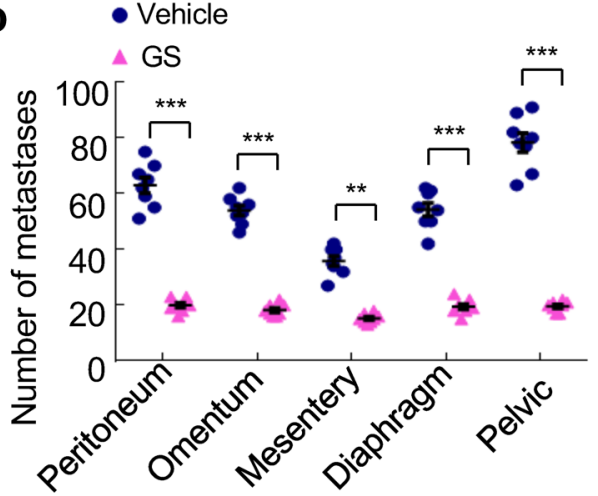

$\mathbf{F}$

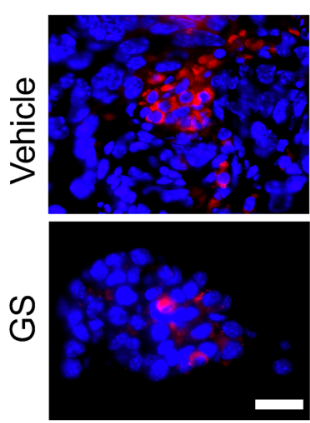

B

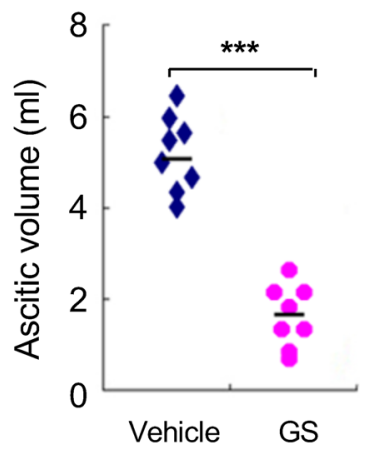

C

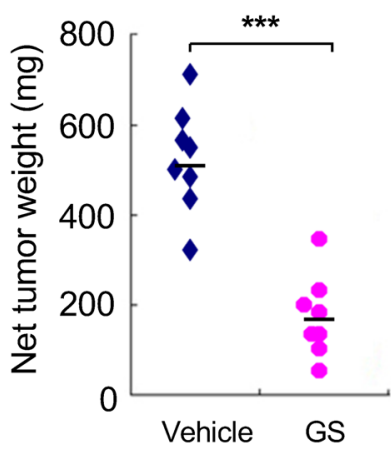

$\mathbf{E}$

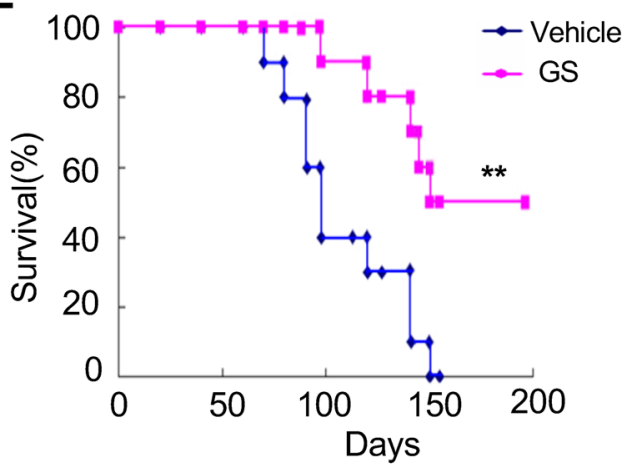

H
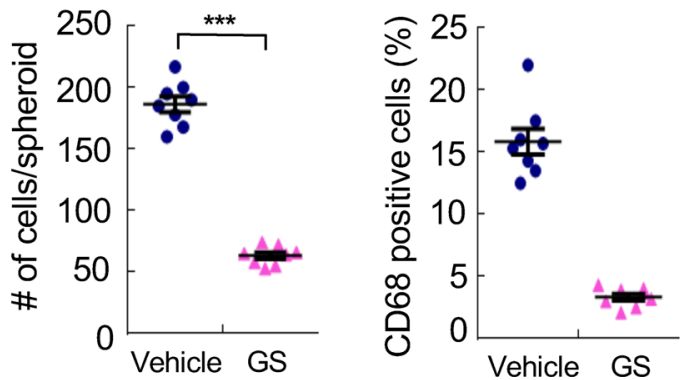

Figure 7. ASK1 inhibitor reduces macrophage infiltration and tumor growth in the mouse models. WT mice were subjected to peritoneal-injection of ID8 cells $\left(1 \times 10^{6}\right)$. Mice were randomly divided into two groups on day 7 after implantation and were fed with vehicle control or ASK1 inhibitor (CS444217) (0.2\% in chow). (A) Body weights were measured every 3 days as indicated, and body weight gains were presented. (B-H) Mice were sacrificed on day 70 after injection of ID8 cells. (B and C) Statistical analyses of ascite volume and tumor weight in vehicle and ASK1 inhibitor groups. Data are presented as means \pm SEM, $n=10$. ${ }^{* *} P<0.001$ (two-sided student's $t$ test). (E) Survival curves of vehicle and ASK1 inhibitor-treated groups after ID8 peritoneal injection. Data are expressed as mean $\pm \mathrm{SEM}, n=22$. ${ }^{* *} P<0.01$. (F-H) Spheroids from ascites were collected and mounted on slides. Spheroids were examined by CD68 staining (F). Scale bar: $20 \mu \mathrm{m}$. Spheroid sizes $(\mathbf{G})$ and $\%$ of CD68 ${ }^{+}$cells within spheroids $(\mathbf{H})$ were quantified. Data are presented as means \pm SEM, $n=10$. ${ }^{* * *} P$ $<0.001$ (two-sided student's $t$ test).

C-D). Similarly, TNF- $\alpha$ alone also induced a complete disruption of EC VE-cadherin junctions by 30-minute treatment of human lung microvascular endothelial cells (HLMVECs) (Figure 6A), with no effect on VE-cadherin mRNA levels (data not shown). Interestingly, we observed a colocalization of VE-cadherin with lysosomal marker Lamp1 upon TNF- $\alpha$ treatment (Figure 6A). Moreover, TNF- $\alpha$-induced VE-cadherin degradation was blocked by lysosomal inhibitor chloroquine, but not by proteasomal inhibitor MG132 (Figure 6, A-D). These data suggest that VE-cadherin is degraded through an endocytosis/lysosome-mediated pathway. Importantly, an ASK1-specific inhibitor GS444217 also completely blocked TNF- $\alpha$-induced VE-cadherin degradation. Of note, an inhibitor of the ASK1 downstream effector JNK (SP600125), but not the p38 inhibitor SB203580, blocked VE-cadherin degradation under the same conditions (Figure 6, A-D). We observed similar effects of ASK1-JNK-lysosome inhibitors on PE-ID8 conditional media-induced VE-cadherin degradation in 
A

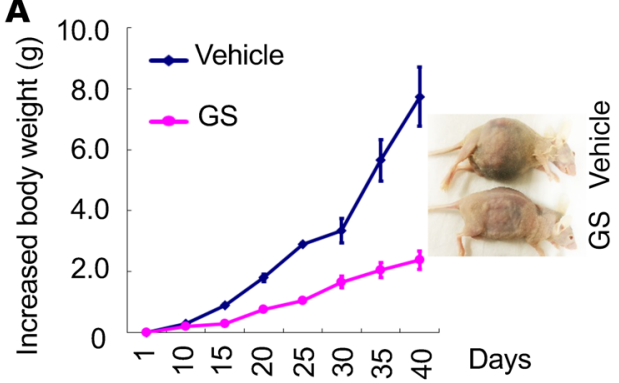

C

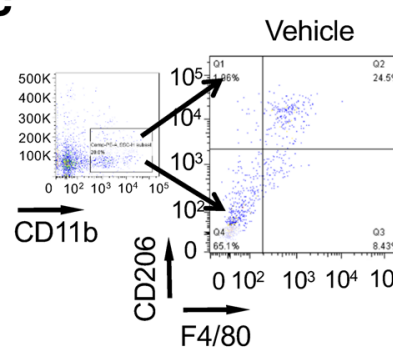

E

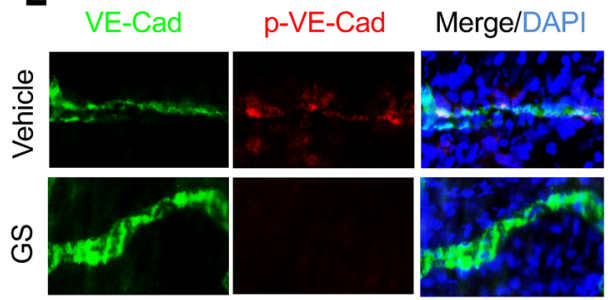

B

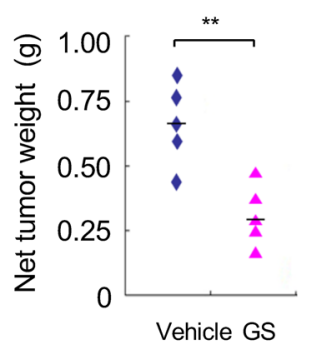

D

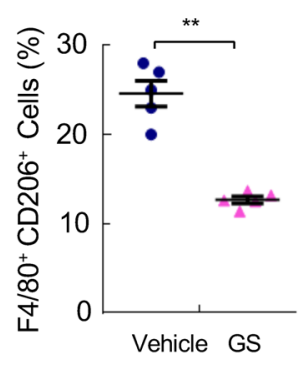

$\mathbf{F}$

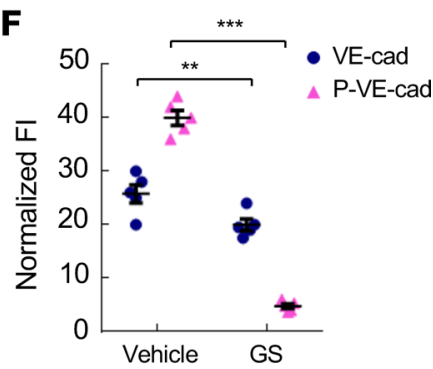

Figure 8. ASK1 inhibitor reduces macrophage infiltration and tumor growth in the xenograft mouse models. An orthotopic ovarian cancer mouse model was established by peritoneal injection of human SKOV3 cells into female recipient nude mice. Mice were then either untreated (vehicle) or treated with ASK inhibitor ( $0.2 \%$ in chow). (A) Mouse body weights were measured at indicated time points. Representative images of mouse bodies in control and ASK1 inhibitor groups. (B) Net tumor weights were measured at day 38. (C) CD11b+F4/80+CD206 ${ }^{+}$ macrophages in the peritoneal cavity were analyzed by FACS. (D) Percentage of CD11b+F4/80+CD206 ${ }^{+}$ macrophages was quantified. (E) Immunoflourecent stainings of diaphragm harvested from the xenograft tumor model. Representative images show costaining of VE-cadherin and $p$-VE-cadherin in diaphragm. Magnification, 630x. (F) Relative $\mathrm{VE}$-cadherin and $\mathrm{p}-\mathrm{VE}$-cadherin levels were quantified. All data are presented as mean \pm SEM, $n=5$ for each group. ${ }^{* *} P<0.01$; ${ }^{* *} P<0.001$ (two-sided student's $t$ test). (C) A model for the role of ASK1 in mediating macrophage infiltration and promoting OC progression. ASK1-JNK activation in peritoneal microvessels mediates VE-cadherin degradation and increases endothelial permeability, facilitating TAM infiltration. Peritoneal TAMs in the peritoneal cavity promote ovarian cancer spheroid formation, tumor growth, and peritoneal implantation. Genetic and pharmacological inhibitions of ASK1 (by the SOCS1 transgenesis, ASK1 deficiency, and ASK1 inhibitor) block TAM infiltration and ovarian cancer growth and implantation.

G
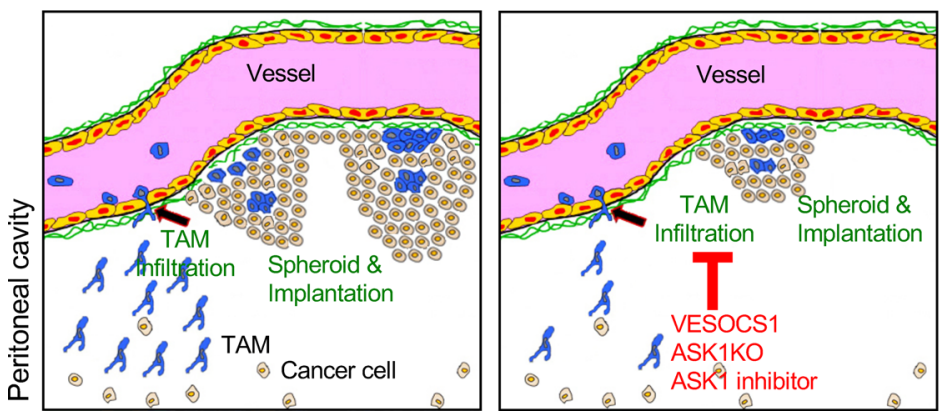

MLMVECs (Supplemental Figure 7). Phosphorylation of VE-cadherin at Tyrosine-658 is a critical step for VE-cadherin degradation (33, 34). Indeed, we detected that ASK1 inhibitor GS444217 drastically inhibited TNF-induced VE-cadherin phosphorylation and degradation in HLMVECs (Figure 6, E and F). Taken together, these data indicate that ASK1 and its downstream effector JNK signaling are critical in mediating the disruptions of VE-cadherin junctions induced by tumor cell-derived cytokines (Figure 6G).

Therapeutic effects of the ASK1 inhibitor on TAM infiltration and ovarian tumor growth in mouse models. To explore a therapeutic effect of the ASK1 inhibitor in treatment of ovarian caner, ID8 tumor-bearing mice were treated with GS444217 in a chow diet, as we performed previously (35). Phenotype analyses showed that GS444217 markedly attenuated accumulation of ascites fluid, tumor growth, and peritoneal implantations (Figure 7, A-D). Accordingly, the survival of tumor-bearing mice was greatly increased by GS444217 treatment compared with the vehicle group (Figure 7E). However, GS444217 had no direct inhibitory effect on proliferation and survival of tumor cells in vitro (Supplemental Figure 8). As observed in ASK1-KO mice and VESOCS1 mice, inhibition of ASK1 by GS444217 stabilized VE-cadherin expression in peritoneal microvessels (Supplemental Figure 9, A and B), with reduced macrophage infiltration into the peritoneal cavity measured by FACS analyses compared with vehicle control groups (Supplemental Figure 10, A and B). Similar to ASK1 deficiency, ASK1 inhibitor GS444217 had no effects on M2 macrophage polarization in the OC model as determined by qRT-PCR for M1- and M2-like macrophage markers (Supplemental Figure 10C). However, the sizes of tumor spheroids and $\mathrm{CD} 68^{+}$macrophages inside the spheroids were markedly reduced by ASK1 inhibitor treatment (Figure 7, F-H). However, the ASK1 inhibitor had no effects on tumor growth, ascites formation, abdominal cavity metastasis, or survival rate in the ID8 OC model using 
ASK1-KO female mice as recipients (Supplemental Figure 11). Taken together, these data suggest that the ASK1 inhibitor, by blocking ASK1-dependent macrophage infiltration, attenuates OC tumor peritoneal implantation and progression.

We also determined the therapeutic effects of the ASK1 inhibitor in an orthotopic mouse model with human SKOV3 cells injection into female recipient nude mice. Similar to the ID8 model, GS444217 markedly attenuated accumulation of tumor growth (Figure 8, A and B). GS444217 reduced macrophage infiltration into the peritoneal cavity, as measured by FACS analyses with F4/80 and CD206 immunostaining (Figure 8, C and D). We also observed that GS444217 reduced p-VE-cadherin concomitant stabilized VE-cadherin expression in peritoneal microvessels (Figure 8, E and F). Taken together, these data suggest that ASK1 plays a critical role in mediating VE-cadherin degradation, macrophage infiltration, and OC progression.

\section{Discussion}

A tight association of TAMs with OC has been established in both mouse models and human patients. Depletion of peritoneal macrophages, but not neutrophils and NK cells, reduces the chance of peritoneal metastasis (36). Recently, we have reported that macrophages play a critical role in OC peritoneal implantation and metastasis by mediating tumor spheroid formation. Our report suggests the following model for a role of macrophage in early stages of OC tumor growth and transcoelomic metastasis (27) (also see Figure $8 \mathrm{G})$. During early stages of OC transcoelomic metastasis and tumor growth, detached OC cells induce infiltration of macrophages into the peritoneal cavity. Interactions of OC cells with infiltrated macrophages in the peritoneal environment form spheroids and skew macrophages into M2 subtype TAMs. Interestingly, TAMs is located in the center of spheroids, where they may provide initial matrix support for OC to avoid anoikis, a form of programmed cell death that occurs in OC cells when they detach from the primary tumor site. We further uncovered that TAM-secreted EGF activates EGFR-VEGFR signaling that is upregulated on tumor cells to promote tumor migration, adhesion, and spheroid formation. Given that ASK1 is critical for macrophage activation and inflammation (20), our initial objective was to investigate the role of ASK1 in TAM-promoted OC progression. As expected, a global deletion of ASK1 attenuated TAM-spheroid formation and OC progression. To our surprise, we found that ASK1 in TAMs was not critical for OC peritoneal growth from reciprocal TAM transplantation assays. Specifically, TAMs from both WT and ASK1KO donor mice similarly augmented tumor growth, accumulation of ascitic fluid, and net tumor weight, as well as tumor implantations in WT recipient mice; ASK1-deficient TAMs also promote tumor growth once implanted into the peritoneal cavity, even in ASK1-KO recipient mice. These results prompted us to examine if ASK1 regulates macrophage infiltration into the peritoneal cavity. We found that ASK1 deficiency drastically attenuated peritoneal macrophage infiltration and TAM-tumor cell spheroid formation without effects on TAM polarization. By using vascular EC-specific transgenic mice expressing the ASK1 suppressor SOCS1 and ASK1-specific pharmacological inhibitors, we demonstrated that ASK1 mediated tumor-induced vascular permeability and macrophage transmigration across the endothelium during early OC growth (Figure 8G). Transcoelomic (peritoneal) metastasis occurs in nearly all patients with OC (more than $90 \%)$ and can also happen in many other cancers, such as pancreatic (50\%) and colon cancers $(32 \%)$ (37). Therefore, our study provides ASK1 as a potentially novel target for the treatment of OC and other transcoelomic metastasis cancers.

Although the importance of macrophages in OC growth has been well studied, a detailed mechanism regarding how tumor cells induce monocyte infiltration into the peritoneal cavity is largely unknown. We observed that very few macrophages were detected in the peritoneal cavity at 2 hours after injection of ID 8 OC cells in the orthotopic OC model. However, macrophages account for $66.3 \%$ of the total peritoneal cells 3 weeks after ID8 injection. Moreover, an increase in tumor cell number at 3 weeks after implantation is correlated with formation of TAM-tumor spheroids and subsequent tumor growth. These results suggest that tumor cells induce macrophage infiltration at early stages of OC progression. This represents a common process for other solid tumors where TAMs are critical components of tumor microenvironments, promoting tumor growth, tumor angiogenesis, immune suppression, metastasis, and chemoresistance ( 38 , 39). Therefore, blocking TAM infiltration into tumor tissues is an emerging target against tumors (40). The efficacy of CSF1R CSF-1 receptor (CSF1R; also known as macrophage CSF receptor MC-SFR or c-Fms kinase) inhibitors in blocking the enhanced uptake of monocytes during paclitaxel treatment in preclinical studies (41) has prompted clinical trials of their use in combination with either paclitaxel or the antiproliferative agent eribulin (http://www.clinicaltrials.gov). Various preclinical studies have also highlighted 
ways to reprogram TAMs from an M2- to an M1-like phenotype in tumors $(4,42,43)$. However, very little is known about strategies in modulations of microvascular permeability to block monocyte transmigration and infiltration into tumor tissues. Our study has identified ASK1, a kinase involved in vascular EC activation and inflammation, as a critical molecule mediating tumor cell-induced macrophage transmigration. Mechanistic studies support the following model for the role of ASK1 in mediating macrophage infiltration and promoting OC progression (Figure 6G and Figure 8G). Specifically, our data support a model in which tumor-derived cytokines activate ASK1 and its downstream effector JNK in peritoneal microvessels. This activation of ASK1/JNK mediate sVE-cadherin degradation and endothelial permeability, facilitating macrophage transmigration. Peritoneal macrophages are polarized into M2-like TAMs and form tumor spheroids, promoting tumor growth and peritoneal implantations. Genetic and pharmacological inhibitions of ASK1 (by the SOCS1 transgenesis, ASK1 deficiency, and ASK1 inhibitor) block vascular permeability, macrophage transmigration, and OC growth and metastasis.

The role of ASK1 in regulation of microvascular permeability, an early step prior to cytokine production by immune cells in tumor tissues, has not been previously investigated. We reason that blockade of vascular permeability and immune cell transmigration would provide an alternative or better approach for treatment of cancer. We found that OC tumor cells were induced to produce proinflammatory cytokines in the peritoneal microenvironment; tumor cell-secreted cytokines (such as TNF) activate the ASK1/ JNK pathway, which in turn induces vascular permeability and macrophage infiltration. This might be important for initial entry of macrophages into the peritoneal cavity; infiltrated macrophages are known to produce abundant proinflammatory cytokines to activate vascular ECs, forming a positive feedback loop. We demonstrated that ASK1 also mediated a peritoneal entry of macrophages in a classical thioglycollate model. Furthermore, we defined a specific pathway involved in ASK1-mediated vascular permeability. Normal microvascular permeability depends on the maintenance of the endothelial barrier by VE-cadherin-associated adherens junctions. VE-cadherin disorganization or/and downregulation has been shown to be an important marker for EC junctional disruption and permeability (44). VE-cadherin and vascular permeability can be regulated by several pathways, including VE-cadherin phosphorylation-dependent and endocytosis-mediated pathways $(33,34)$. We showed that tumor-derived conditional media (and cytokine TNF) strongly induced VE-cadherin endocytosis and degradation via a lysosome-dependent pathway, and the VE-cadherin degradation and EC junctional disruption were blocked by ASK1- and JNK-specific inhibitors. Most importantly, a selective ASK1 inhibitor GS444217 attenuated macrophage infiltration, TAM-tumor spheroid formation, and tumor growth in the orthotopic mouse models. However GS444217 has no effects on OC progression in the ASK1-KO female recipient mice, supporting a specific effect of GS444217 on ASK1-mediated processes.

Taken together, our study demonstrates that TAMs play an essential role in tumor growth during the process of transcoelomic metastasis of OC. SOCS1 functions as a negative regulator in TNF-induced inflammation and ASK1 degradation in EC. The activated ASK1-JNK signaling can promote VE-cadherin degradation. Inhibition of VE-cadherin degradation blocked macrophage infiltration into tumor tissue. Our current study has unveiled the underlying mechanism of interaction of microvessels with tumor growth, providing a strategy to inhibit the implantation metastasis and improve the prognosis of OC patients.

\section{Methods}

Animal model. All animal studies were approved by the IACUC of Yale University. All mice were maintained in a C57BL/6 background. ASK1-KO mice were obtained from H. Ichijo from University of Tokyo (Tokyo, Japan) (20). Myeloid-specific Cre deleter (LysMCre) strain (B6.129P2-Lyz2tm1(cre)Ifo/J) was purchased from The Jackson Laboratory. The Cre-knock-in allele has a Cre recombinase inserted into the lysozyme 2 gene, both abolishing endogenous Lyz2 gene function and placing NLS-Cre expression under the control of the endogenous Lyz2 promoter/enhancer elements. LysMCre mice have been widely used for Cre-lox studies of the myeloid cell lineage. LysMCre mice were crossed with tomato reporter mice to express GFP in myeloid lineage cells (monocyte/neutrophils). Light intensity inside the cages ranged from 100-200 lux under a 12-hour light/dark cycle.

Mouse OC ID8 or SKOV3 $\left(1 \times 10^{6} / \mathrm{ml}\right)$ were injected into the abdominal cavity of C57BL/6 background or nude mice in $100 \mu 1$ of DMEM. Mice body weight gain, ascitic fluid volume, and tumor weight were measured by electronic balance. The mice were sacrificed after 60 days, and the tumor spheroids and tumor implantations were analyzed by histology. 
Administration of ASK1 inhibitor (ASK1i) GS444217 in mice. The tumor-bearing mice were administered ASK1i ad libitum in regular chow $(0.2 \%$ GS444217) as indicated. Vehicle groups receiving normal chow were used as controls (35).

Generation of the vascular endothelial SOCS1 transgenic mouse. The VE-cadherin promoter in a TA vector was obtained from Laura Benjamin (Harvard Medical School, Boston, Massachusetts, USA). The 3'-UTR (untranslated region) from bovine growth hormone $(\mathrm{bGH})$ was cloned downstream of the VE-cadherin promoter to generate the pVE-pA vector. The human SOCS1 cDNA with a Myc-tag sequence at the 3'-end was inserted into the EcoRI and $X b a I$ sites between the VE-cadherin promoter and bGH pA to obtain a pVE-SOCS1 plasmid. The plasmid was linearized by XhoI digestion, and the pronuclear injection was performed at the Yale Transgenic Core. The founder transgenics were identified by PCR of tail DNA with a $5^{\prime}$ primer of Trx2 and 3' primer of Myc. VESOCS1 transgenic mice were backcrossed with C57BL/6 mice for more than 10 generations before experiments.

In vivo treatment in mouse models. Mice were divided into 10 mice per group. An orthotopic mouse model was established by injecting mouse ID8 OCs i.p. to C57BL/ 6 female recipient mice. WT mice were subjected to peritoneal-injection of ID8 cells $\left(1 \times 10^{6}\right)$. Mice were randomly divided into two groups on day 7 after implantation and were fed with ASK1 inhibitor (GS444217) and vehicle control, respectively. Experimental and control mice were killed 50 days after injection of tumor cells. We collected ascites and spheroid for $\mathrm{H} \& \mathrm{E}$ staining and FACS.

Evans blue permeability assay. Five days after viral administration, Evans blue dye (100 $\mu 1$ of a $1 \%$ solution in $0.9 \% \mathrm{NaCl}$; Sigma-Aldrich) was injected into the retro-orbital plexus of anesthetized WT, ASK1KO, and VESOCS1 mice. Thirty minutes after the injection, mice were sacrificed and perfused with PBS through the left ventricle to clear the dye from the vascular volume. Ears were removed, dried in $60^{\circ} \mathrm{C}$ overnight, and weighed before Evans blue extraction using $1 \mathrm{ml}$ formamide at $55^{\circ} \mathrm{C}$ for 16 hours. Evans blue content was quantified by reading at $630 \mathrm{~nm}$ in a spectrophotometer as described previously (SmartSpec Plus Spectrophotometer, Bio-Rad) $(45,46)$.

Cell culture. The ID8 (mouse epithelial OC line) was gifted from Jack Lawler and Carmelo Nucera at Beth Israel Deaconess Medical Center, Harvard Medical School (47). SKOV3 (human ovarian adenocarcinoma cell line) were obtained from ATCC. SK-OV-3 cells are resistant to tumor necrosis factor and to several cytotoxic drugs, including diphtheria toxin, cisplatinum, and adriamycin. ID 8 and SKOV3 cells were cultured in DMEM (Invitrogen) supplemented with $10 \% \mathrm{FBS}, 100 \mathrm{U} / \mathrm{ml}$ penicillin, and $100 \mu \mathrm{g} / \mathrm{ml}$ streptomycin at $37^{\circ} \mathrm{C}$ in a humidified atmosphere of $5 \% \mathrm{CO}_{2}$ and $95 \%$ air. The culture medium was changed every 2 days, and cells were split when they reached $80 \%-90 \%$ confluence. For all experiments, cells were seeded at an appropriate density and grew to $80 \%-90 \%$ confluence before experimentation. Primary mouse TAMs and ID8 cells within the peritoneal spheroids (PE-ID8) were isolated from the peritoneal cavity after i.p. injection of ID8 cells for different time points in C57BL/6-background mice. Primary HLMVEC were purchased from Angio-Proteomie. Isolation of primary vascular ECs from mouse lung tissues was performed as described previously $(48,49)$.

Recombinant protein and inhibitors. Recombinant human and mouse TNF were purchased from R\&D Systems. EGFR inhibitor was purchased from Roche. SP600125 (JNK inhibitor), SB203580 (MAPK inhibitor), and MG132 were purchased from EMD Millipore. GS444217 (ASK1 inhibitor) was purchased from Gilead Company. Chloroquine was purchased from Sigma-Aldrich.

Immunostaining. Antibodies used for IHC and immunofluorescent staining are listed in Supplemental Table 1. Confocal microscopy images were taken with a Zeiss-LSM 700 microscope and evaluated using the ZEN2010 software. For mean fluorescence intensity measurements, confocal microscopy images were analyzed with ImageJ (NIH). Slides were observed using a Zeiss Axiovert 200 fluorescence microscope (Carl Zeiss MicroImaging), and images were captured using Openlab3 software (Improvision). For tissue, 5 - $\mu \mathrm{m}$ serial sections cut from frozen, OCT-embedded tissues were fixed in $-20^{\circ} \mathrm{C}$ acetone for 10 minutes and dried for 15 minutes, followed by the same blocking/antibody protocol for cells as listed above.

$q R T-P C R$. Total RNA was extracted from human tissues using the RNeasy Plus Mini Kit (74134, Qiagen), and then converted into cDNAs using the High Capacity cDNA Reverse Transcription Kit (4368814, Applied Biosystems) following the manufacturer's instruction. qPCR was performed with a CFX-96 (Bio-Rad) using the RT2 SYBR Green (330500, SA Biosciences). All primers used for qRT-PCR are listed in Supplemental Table 2. All values were normalized with GAPDH abundance. Data were presented as the average of triplicates $\pm \mathrm{SD}$. 
Protein extraction and Western blot analysis. Freshly dissected unfixed tissue was homogenized in lysis buffer. The lysates were centrifuged at $13,000 \mathrm{~g}$ for 10 minutes at $4^{\circ} \mathrm{C}$. Supernatants were collected and determined with a Bradford Protein Assay kit (Bio-Rad). The cell lysates were subjected to SDS-PAGE followed by immunoblotting (Immobilon P; Millipore) with specific antibodies followed by detection using an enhanced chemiluminescence kit (Amersham Life Science). All antibodies used for Western blotting are listed in Supplemental Table 1.

FACS. Analyses of mouse cell surface CD11b, F4/80, CD3e, CD206, and CD163 and human cell surface CD14 and CD326 expression by FACS were described previously (4, 5, 50-52). Briefly, mouse TAMs suspensions were stained with mouse CD11b-FITC, F4/80-PE, CD3e-APC, PE-CD206, and APC-CD163 and human CD14 and CD326 antibodies for 15 minutes on ice. Isotype antibody serves as negative control. Flow cytometry was performed on a FACSCalibur (BD Biosciences). Data were analyzed with BD Cell Quest Pro software. All antibodies used for FACS are listed in Supplemental Table 1.

Transwell migration assay and transwell coculture. Cell migration was examined using Transwell fitted with polycarbonate filters (8-um pore size) (Corning Inc.). Briefly, the under-surfaces of the filters were coated overnight at $4^{\circ} \mathrm{C}$ with fibronectin $(20 \mu \mathrm{g} / \mathrm{ml})$ in PBS ( $\mathrm{pH}$ 7.4). The coating solution was removed from the lower chamber before filling with FBS free medium. MLMVECs were seeded at the top of the transwell (Figure 5A), and tumor cells (ID8 cells) were seeded at the bottom well of the Boyden Chamber at 12 hours, but MLMVEC and tumor cells were completely separate. After 12 hours, monocytes (GFP) were seeded at the top of the transwell (Figure 5A). The three types of cells were grown without direct cell-to-cell contact. We observed the number of monocytes infiltration from top well to bottom well by fluorescence microscope (Carl Zeiss Axiostar Plus Microscope).

Statistics. Group sizes were determined by an a priori power analysis for a two-tailed, two-sample $t$ test with an $\alpha$ of 0.05 and power of 0.8 , in order to detect a $10 \%$ difference in lesion size at the endpoint. Animals were grouped with no blinding but were randomized during the experiments. Female animals were used for all experiments because of OC models. No samples or animals were excluded from analysis. The differences between results of Western blot, qRT-PCR, cell proliferation assay, immunostainings, FACS, and tumor growth were analyzed by student $t$ test. The differences of demographic characteristics of OC patients were analyzed by $\chi^{2}$ or Fisher's exact test. Kaplan-Meier method was used to estimate overall survival (OS), while the difference in the levels among possible prognostic factors was compared by the log-rank test with univariate analyses. A multivariate Cox regression (proportional hazard model) was employed to identify prognostic factors and evaluate the independent impact of CD68 level on OS. Statistical analyses in this study were performed using SAS software (version 9.1.4, SAS Institute). All statistical tests were two-tailed, and $P$ values less than 0.05 were considered statistically significant.

Study approval. Mice were cared for in accordance with NIH guidelines, and all procedures were approved by the Yale University Animal Care and Use Committee. No human subjects were used in this study.

\section{Author contributions}

MY, HJZ, JZ, CL, HL, XL, YL, HZ, and WJ performed experiments. MY and WM designed and analyzed experiments and wrote the manuscript. DGB was involved in the design of experiments and edited the manuscript.

\section{Acknowledgments}

This work was supported by National Key Research and Development Program of China (2016YFC1300600), National Natural Science Foundation of China (91539110, U1601219, and 81371019) and Scientific Grants of Guangdong (2015B020225002 and 2015A050502018). This work was partly supported by NIH grants R01 HL109420 and HL115148, and by a grant from Gilead. Research reported in this publication was partly supported by the NIDDK under NIH award number U54DK106857.

Address correspondence to: Wang Min, Interdepartmental Program in Vascular Biology and Therapeutics, Department of Pathology, Yale University School of Medicine, 10 Amistad Street, 401B, New Haven, Connecticut 06520, USA. Phone: 203.785.6047; Email: wang.min@yale.edu. 
1. Kerbel RS. Tumor angiogenesis. NEngl J Med. 2008;358(19):2039-2049.

2. Condeelis J, Pollard JW. Macrophages: obligate partners for tumor cell migration, invasion, and metastasis. Cell. 2006;124(2):263-266.

3. Qian BZ, Pollard JW. Macrophage diversity enhances tumor progression and metastasis. Cell. 2010;141(1):39-51.

4. Hagemann T, et al. Ovarian cancer cells polarize macrophages toward a tumor-associated phenotype. J Immunol. 2006;176(8):5023-5032.

5. Ko SY, Ladanyi A, Lengyel E, Naora H. Expression of the homeobox gene HOXA9 in ovarian cancer induces peritoneal macrophages to acquire an M2 tumor-promoting phenotype. Am J Pathol. 2014;184(1):271-281.

6. Steidl C, et al. Tumor-associated macrophages and survival in classic Hodgkin's lymphoma. N Engl J Med. 2010;362(10):875-885.

7. De Palma M, Lewis CE. Macrophage regulation of tumor responses to anticancer therapies. Cancer Cell. 2013;23(3):277-286.

8. Ichijo H, et al. Induction of apoptosis by ASK1, a mammalian MAPKKK that activates SAPK/JNK and p38 signaling pathways. Science. 1997;275(5296):90-94.

9. Tobiume K, et al. ASK1 is required for sustained activations of JNK/p38 MAP kinases and apoptosis. EMBO Rep. 2001;2(3):222-228.

10. Pober JS, Min W, Bradley JR. Mechanisms of endothelial dysfunction, injury, and death. Annu Rev Pathol. 2009;4:71-95.

11. Chen J, Fujii K, Zhang L, Roberts T, Fu H. Raf-1 promotes cell survival by antagonizing apoptosis signal-regulating kinase 1 through a MEK-ERK independent mechanism. Proc Natl Acad Sci USA. 2001;98(14):7783-7788.

12. Kim AH, Khursigara G, Sun X, Franke TF, Chao MV. Akt phosphorylates and negatively regulates apoptosis signal-regulating kinase 1. Mol Cell Biol. 2001;21(3):893-901.

13. Zhang R, et al. Hsp90-Akt phosphorylates ASK1 and inhibits ASK1-mediated apoptosis. Oncogene. 2005;24(24):3954-3963.

14. Iriyama T, et al. ASK1 and ASK2 differentially regulate the counteracting roles of apoptosis and inflammation in tumorigenesis. EMBO J. 2009;28(7):843-853.

15. Osaka N, et al. ASK1-dependent recruitment and activation of macrophages induce hair growth in skin wounds. J Cell Biol. 2007;176(7):903-909.

16. Wang TH, et al. Microtubule dysfunction induced by paclitaxel initiates apoptosis through both c-Jun N-terminal kinase (JNK)-dependent and -independent pathways in ovarian cancer cells. J Biol Chem. 1999;274(12):8208-8216.

17. Ji W, et al. Carbamoylating activity associated with the activation of the antitumor agent laromustine inhibits angiogenesis by inducing ASK1-dependent endothelial cell death. PLoS ONE. 2014;9(7):e103224.

18. Liu Y, Min W. Thioredoxin promotes ASK1 ubiquitination and degradation to inhibit ASK1-mediated apoptosis in a redox activity-independent manner. Circ Res. 2002;90(12):1259-1266.

19. Liu Y, Yin G, Surapisitchat J, Berk BC, Min W. Laminar flow inhibits TNF-induced ASK1 activation by preventing dissociation of ASK1 from its inhibitor 14-3-3. J Clin Invest. 2001;107(7):917-923.

20. Matsuzawa A, et al. ROS-dependent activation of the TRAF6-ASK1-p38 pathway is selectively required for TLR4-mediated innate immunity. Nat Immunol. 2005;6(6):587-592.

21. Chiang E, Dang O, Anderson K, Matsuzawa A, Ichijo H, David M. Cutting edge: apoptosis-regulating signal kinase 1 is required for reactive oxygen species-mediated activation of IFN regulatory factor 3 by lipopolysaccharide. J Immunol. 2006;176(10):5720-5724.

22. Jemal A, Siegel R, Ward E, Hao Y, Xu J, Thun MJ. Cancer statistics, 2009. CA Cancer J Clin. 2009;59(4):225-249.

23. Siegel R, Naishadham D, Jemal A. Cancer statistics, 2012. CA Cancer J Clin. 2012;62(1):10-29.

24. Tan DS, Agarwal R, Kaye SB. Mechanisms of transcoelomic metastasis in ovarian cancer. Lancet Oncol. 2006;7(11):925-934

25. Peart T, et al. Intact LKB1 activity is required for survival of dormant ovarian cancer spheroids. Oncotarget. 2015;6(26):22424-22438.

26. Rafehi S, et al. TGF $\beta$ signaling regulates epithelial-mesenchymal plasticity in ovarian cancer ascites-derived spheroids. Endocr Relat Cancer. 2016;23(3):147-159.

27. Yin M, et al. Tumor-associated macrophages drive spheroid formation during early transcoelomic metastasis of ovarian cancer. J Clin Invest. 2016;126(11):4157-4173.

28. He Y, Zhang W, Zhang R, Zhang H, Min W. SOCS1 inhibits tumor necrosis factor-induced activation of ASK1-JNK inflammatory signaling by mediating ASK1 degradation. J Biol Chem. 2006;281(9):5559-5566

29. Yu L, et al. JAK2 and SHP2 reciprocally regulate tyrosine phosphorylation and stability of proapoptotic protein ASK1. J Biol Chem. 2009;284(20):13481-13488.

30. Qin L, et al. SOCS1 prevents graft arteriosclerosis by preserving endothelial cell function. J Am Coll Cardiol. 2014;63(1):21-29.

31. Krebs DL, Hilton DJ. SOCS: physiological suppressors of cytokine signaling. J Cell Sci. 2000;113 (Pt 16):2813-2819.

32. Zhang X, Goncalves R, Mosser DM. The isolation and characterization of murine macrophages. Curr Protoc Immunol. 2008; Chapter 14:Unit 14.1.

33. Sun Z, et al. VEGFR2 induces c-Src signaling and vascular permeability in vivo via the adaptor protein TSAd. $J$ Exp Med. 2012;209(7):1363-1377.

34. Eliceiri BP, Paul R, Schwartzberg PL, Hood JD, Leng J, Cheresh DA. Selective requirement for Src kinases during VEGFinduced angiogenesis and vascular permeability. Mol Cell. 1999;4(6):915-924.

35. Huang Q, et al. Thioredoxin-2 inhibits mitochondrial reactive oxygen species generation and apoptosis stress kinase-1 activity to maintain cardiac function. Circulation. 2015;131(12):1082-1097.

36. Robinson-Smith TM, et al. Macrophages mediate inflammation-enhanced metastasis of ovarian tumors in mice. Cancer Res. 2007;67(12):5708-5716

37. Lu Z, Wang J, Wientjes MG, Au JL. Intraperitoneal therapy for peritoneal cancer. Future Oncol. 2010;6(10):1625-1641.

38. Rolny C, et al. HRG inhibits tumor growth and metastasis by inducing macrophage polarization and vessel normalization through downregulation of PIGF. Cancer Cell. 2011;19(1):31-44.

39. Franklin RA, et al. The cellular and molecular origin of tumor-associated macrophages. Science. 2014;344(6186):921-925.

40. Huang Y, Snuderl M, Jain RK. Polarization of tumor-associated macrophages: a novel strategy for vascular normalization and antitumor immunity. Cancer Cell. 2011;19(1):1-2.

41. Pyonteck SM, et al. CSF-1R inhibition alters macrophage polarization and blocks glioma progression. Nat Med. 
2013;19(10):1264-1272.

42. DeNardo DG, et al. Leukocyte complexity predicts breast cancer survival and functionally regulates response to chemotherapy. Cancer Discov. 2011;1(1):54-67.

43. Guiducci C, Vicari AP, Sangaletti S, Trinchieri G, Colombo MP. Redirecting in vivo elicited tumor infiltrating macrophages and dendritic cells towards tumor rejection. Cancer Res. 2005;65(8):3437-3446.

44. Dejana E, Tournier-Lasserve E, Weinstein BM. The control of vascular integrity by endothelial cell junctions: molecular basis and pathological implications. Dev Cell. 2009;16(2):209-221.

45. Miles AA, Miles EM. Vascular reactions to histamine, histamine-liberator and leukotaxine in the skin of guinea-pigs. J Physiol (Lond). 1952;118(2):228-257.

46. Thurston G, et al. Leakage-resistant blood vessels in mice transgenically overexpressing angiopoietin-1. Science. 1999;286(5449):2511-2514.

47. Russell S, Duquette M, Liu J, Drapkin R, Lawler J, Petrik J. Combined therapy with thrombospondin-1 type I repeats (3TSR) and chemotherapy induces regression and significantly improves survival in a preclinical model of advanced stage epithelial ovarian cancer. FASEB J. 2015;29(2):576-588.

48. Luo D, et al. AIP1 is critical in transducing IRE1-mediated endoplasmic reticulum stress response. J Biol Chem. 2008;283(18):11905-11912.

49. Zhang H, et al. AIP1 functions as an endogenous inhibitor of VEGFR2-mediated signaling and inflammatory angiogenesis in mice. J Clin Invest. 2008;118(12):3904-3916.

50. Gordon IO, Freedman RS. Defective antitumor function of monocyte-derived macrophages from epithelial ovarian cancer patients. Clin Cancer Res. 2006;12(5):1515-1524.

51. Duraiswamy J, Freeman GJ, Coukos G. Therapeutic PD-1 pathway blockade augments with other modalities of immunotherapy T-cell function to prevent immune decline in ovarian cancer. Cancer Res. 2013;73(23):6900-6912.

52. Motz GT, Coukos G. Deciphering and reversing tumor immune suppression. Immunity. 2013;39(1):61-73. 